\title{
Phage-Mediated Immune Evasion and Transmission of Livestock- Associated Methicillin-Resistant Staphylococcus aureus in Humans
}

\author{
Raphael N. Sieber, Tinna R. Urth, Andreas Petersen, Camilla H. Møller, Lance B. Price, \\ Robert L. Skov, Anders R. Larsen, Marc Stegger, ${ }^{1}$ and Jesper Larsen ${ }^{1}$
}

\footnotetext{
Medscape ACTIVITY

In support of improving patient care, this activity has been planned and implemented by Medscape, LLC and Emerging Infectious Diseases. Medscape, LLC is jointly accredited by the Accreditation Council for Continuing Medical Education (ACCME), the Accreditation Council for Pharmacy Education (ACPE), and the American Nurses Credentialing Center (ANCC), to provide continuing education for the healthcare team.

Medscape, LLC designates this Journal-based CME activity for a maximum of 1.00 AMA PRA Category 1 Credit(s) ${ }^{\mathrm{TM}}$ Physicians should claim only the credit commensurate with the extent of their participation in the activity.

Successful completion of this CME activity, which includes participation in the evaluation component, enables the participant to earn up to $1.0 \mathrm{MOC}$ points in the American Board of Internal Medicine's (ABIM) Maintenance of Certification (MOC) program. Participants will earn MOC points equivalent to the amount of CME credits claimed for the activity. It is the CME activity provider's responsibility to submit participant completion information to ACCME for the purpose of granting ABIM MOC credit.

All other clinicians completing this activity will be issued a certificate of participation. To participate in this journal CME activity: (1) review the learning objectives and author disclosures; (2) study the education content; (3) take the post-test with a $75 \%$ minimum passing score and complete the evaluation at http://www.medscape.org/journal/eid; and (4) view/print certificate. For CME questions, see page 2796.

Release date: October 16, 2020; Expiration date: October 16, 2021

\section{Learning Objectives}

Upon completion of this activity, participants will be able to:

- Describe the impact of IEC- and tarP-harboring phages on household transmission of LA-MRSA in the North Denmark Region during 2004-2011, according to whole-genome sequencing and epidemiologic investigations

- Determine the association of IEC- and tarP-harboring phages in LA-MRSA in the North Denmark Region during 2004-2011 with spread in the general population, according to an analysis of all Danish patients who had an episode of LA-MRSA infection during 2007-2018

- Identify clinical and public health implications of the effect of IEC- and tarP-harboring phages on household transmission of LA-MRSA in the North Denmark Region during 2004-2011, and of their association with spread into the general population

\section{CME Editor}

Dana C. Dolan, BS, Copyeditor, Emerging Infectious Diseases. Disclosure: Dana C. Dolan, BS, has disclosed no relevant financial relationships.

\section{CME Author}

Laurie Barclay, MD, freelance writer and reviewer, Medscape, LLC. Disclosure: Laurie Barclay, MD, has disclosed no relevant financial relationships.

\section{Authors}

Disclosures: Raphael N. Sieber, PhD; Tinna Urth, MPH; Andreas Petersen, PhD; Camilla Møller, MD, PhD; Lance B. Price, PhD; Robert L. Skov, MD; Anders Larsen, PhD; Marc Stegger, PhD; and Jesper Larsen, PhD, have disclosed no relevant financial relationships.
}

Author affiliations: Statens Serum Institut, Copenhagen, Denmark (R.N. Sieber, T.R. Urth, A. Petersen, C.H. Møller, R.L. Skov, A.R. Larsen, M. Stegger, J. Larsen); Translational Genomics Research Institute, Flagstaff, Arizona, USA
(L.B. Price, M. Stegger); George Washington University, Washington, DC, USA (L.B. Price)

DOI: https://doi.org/10.3201/eid2611.201442

${ }^{1}$ These senior authors contributed equally to this article. 
Livestock-associated methicillin-resistant Staphylococcus aureus (LA-MRSA) can acquire phage-encoded immune modulators, such as the immune evasion cluster (IEC), which protects bacteria from components of the human innate immune system, and the enzyme TarP, which protects against antibody-mediated immune recognition. We used whole-genome sequencing and epidemiologic investigations to study the effects of IEC- and tarP-harboring phages on household transmission of LA-MRSA in North Denmark Region during 2004-2011. We reviewed information about all patients throughout Denmark who experienced LA-MRSA infection during 2007-2018 to determine whether IEC is associated with increased spread into the general population. Horizontal acquisition of IEC in the human host was associated with increased household transmission of LA-MRSA and spillover into the community and healthcare settings, whereas we found no evidence to suggest that IEC-positive LA-MRSA isolates have become self-sustainable in the general population. By contrast, TarP did not seem to influence household transmission of LA-MRSA.

工 ivestock-associated methicillin-resistant Staphylococcus aureus (LA-MRSA) clonal complex (CC) 398 is a major cause of zoonotic disease in Denmark and several other countries in Europe that have industrial pig production (1-3). In Denmark, the prevalence of LA-MRSA CC398 in pig farms increased from 3.5\% in 2008 to $\approx 90 \%$ in 2018 , when LA-MRSA CC398 accounted for $21 \%$ of all human MRSA infections (3). Most LA-MRSA CC398 infections occur in young and otherwise healthy livestock workers and their household contacts (3-5). Although LA-MRSA CC398 seems to be poorly adapted for human-to-human transmission (6), it is nonetheless able to spread to and cause serious illness and even death in elderly and immunocompromised persons in community and healthcare settings (3-5).

S. aureus uses a diverse range of immune-evasive strategies to maintain a lifelong relationship with the human host, many of which are encoded on phages and other mobile genetic elements (7). Of note, human $S$. aureus clones harbor a genetic element, the immune evasion cluster (IEC), on a 44-kb ФSa3int prophage that is stably integrated into the $h l b$ gene on the bacterial chromosome $(8,9)$. The IEC element encodes $\geq 1$ immune modulators, including staphylococcal complement inhibitor, chemotaxis inhibitory protein of staphylococci, staphylokinase, staphylococcal enterotoxin $\mathrm{A}$, and staphylococcal enterotoxin $\mathrm{P}$, which interact specifically with components of the human innate immune system (7). LA-MRSA CC398 isolates are descendants of a human variant of $S$. aureus CC398 but have lost the $\Phi S a 3 i n t$ prophage and the associated IEC element in connection with the host switch event (10); this change provides a potential explanation for the observed relatively low human-to-human transmissibility of LA-MRSA CC398 (6). However, some studies have shown that LA-MRSA CC398 might be capable of readapting to the human host through acquisition of phage-encoded immune modulators. For example, a Denmark study showed that $6 \%$ of human LA-MRSA CC398 isolates collected during 2004-2011 harbored the IEC element, whereas a more recent study revealed that $40 \%$ of LA-MRSA CC398 isolates from pigs in Denmark produce another phage-encoded immune modulator enzyme known as TarP, which enables $S$. aureus to subvert antibody-mediated immune recognition by altering a dominant cell surface epitope known as wall teichoic acids (WTA) $(3,11)$.

These earlier findings raise important questions about the source and dynamics of phages encoding IEC and TarP in LA-MRSA CC398 and their role in host adaptation. In this study, we sequenced and compared a collection of epidemiologically wellcharacterized LA-MRSA CC398 isolates from humans and pigs in North Denmark Region to determine their population structure and the contribution of IEC and TarP to household transmission. We also used national surveillance data to further investigate whether IEC plays a role during spread of LA-MRSA CC398 into the community and healthcare settings.

Data used in this study were collected as part of the national MRSA surveillance program, as approved by the Danish Data Protection Agency (protocol no. 2001-14-0021). The National Committee on Health Research Ethics waived the need for approval and informed consent because data and biologic material were fully anonymized and collected in compliance with national legislation on statutory notification of MRSA in humans.

\section{Methods}

\section{Study Population}

North Denmark Region $\left(\approx 7,900 \mathrm{~km}^{2}\right)$ is a semirural area in northwest Denmark that had a population of $\approx 580,000$ persons and $\approx 3$ million pigs in 2011 (http://www.statbank.dk). We identified the study population using surveillance data collected by the regional infection control staff in North Denmark Region; it included all patients colonized or infected with LA-MRSA CC398 during 2004-2011, as well as all their household contacts who tested negative during the same period. We interviewed all of these persons to obtain relevant information, including sex, age, livestock contact, 
residential address, and workplace, and assigned each to 1 of 3 categories: livestock-exposed persons (direct contact); household contacts of livestock-exposed persons (indirect contact); and persons not connected to livestock production (no contact). We defined livestockexposed persons as primary cases in their households, and the index person as the primary case in households with no connection to livestock production.

\section{Study Isolates and Data Aggregation}

The study isolates comprised 96 human LA-MRSA CC398 isolates from North Denmark Region collected by regional infection control staff during 2004-2011 and 45 LA-MRSA CC398 isolates collected from pigs (1 isolate per farm) in North Denmark Region in 2014 (Appendix 1 Table 1, https://wwwnc.cdc.gov/EID/ article/26/11/20-1442-App1.xlsx). The human isolates have been characterized previously for spa type, presence of IEC, and antimicrobial susceptibilities (3); the pig isolates originated from a study investigating the population structure and dynamics of LA-MRSA CC398 in the pig population in Denmark (12). We used whole-genome sequencing and bioinformatics analyses to study the phylogenetic distribution, genetic diversity, and host association of IEC-harboring and $\operatorname{tar} P$-harboring prophages among the 141 study isolates (Appendix 2, https://wwwnc.cdc.gov/EID/ article/26/11/20-1442-App2.pdf).

\section{Analysis of National Surveillance Data}

MRSA has been notifiable in Denmark since November 2006. As part of the national MRSA surveillance program, local clinical microbiology departments perform $S$. aureus identification and antimicrobial susceptibility testing and submit all confirmed MRSA isolates to the National Reference Laboratory for Antimicrobial Resistance at Statens Serum Institut (Copenhagen, Denmark), which collects patient information from general practitioners and assesses the spa type or the clonal complex and the presence or absence of the IEC element. The following data are collected for each case: sex, age, livestock contact, residential address, indication for testing (screening or infection), and hospitalization dates. Cases without direct or indirect livestock contact are defined as healthcare-onset $(\mathrm{HO})$ if the culture is obtained $\geq 48$ hours after admission; healthcareassociated community-onset (HACO) if the person has had contact with the healthcare setting within the preceding 12 months or the culture is obtained within the first 48 hours after admission; or community-onset (CO) if no other criteria are met. For this study, we retrieved the following information about all patients in Denmark who had an episode of LA-MRSA CC398 infection during January 2007-December 2018 (n = 1,545): sex; age; direct, indirect, or no livestock contact; location of disease onset (e.g., $\mathrm{HO}, \mathrm{HACO}$, or $\mathrm{CO}$ ); and presence or absence of the IEC element in the corresponding LA-MRSA CC398 isolate. We calculated the excess number of clinical cases due to increased spread of IEC-positive isolates into a given patient group of interest as the total number of cases in the patient group of interest multiplied by the difference between the proportion of IEC-positive isolates in the patient group of interest (the sink) and the patient group with direct contact to livestock (the source).

\section{Statistical Analysis}

We used Fisher exact test to analyze categorical data and Student $t$ test to analyze continuous data (GraphPad Prism version 5; GraphPad, https://www. graphpad.com). We reported prevalence differences between different groups as prevalence ratios (PRs) and $95 \%$ CIs. The significance level was set at $\alpha=0.05$.

\section{Results}

\section{Study Population}

A total of 96 patients were colonized or infected with LA-MRSA CC398 in North Denmark Region during 2004-2011, including 67 primary cases and 29 secondary cases from 65 households. A total of 71 household contacts tested negative. The 67 primary cases comprised 57 persons with direct animal contact, 2 with indirect animal contact, and 8 with no animal contact. Those with direct animal contact included 44 pig farm employees from 42 households and 23 animal farms ( 2 households each contained 2 pig farm employees), 3 mink farm employees from 3 households and animal farms, 1 cattle farm employee, 1 turkey farm employee, 1 pig veterinarian, 3 lorry drivers transporting pigs from 3 households, 2 pig abattoir workers from 2 households, and 2 craftsmen working in pig stables from 2 households. Persons with indirect contact were from 2 households (a wife and a child of pig farm employees who were never tested), and those with no contact were from 8 households.

\section{Distribution of IEC and tarP in LA-MRSA CC398}

Most of the human isolates collected from persons living in the same household clustered together, with an average pairwise single-nucleotide polymorphism (SNP) distance of 5.9 (range 0-18 SNPs) and were genotypically homogeneous with respect to presence of specific IEC-harboring and tarP-harboring prophages (Appendix 2 Figure). Furthermore, human isolates from different households connected to the same animal farm 
also tended to cluster together but were genotypically more diverse than isolates from the same household (19.6 SNPs [range 0-156 SNPs] in different households versus 6.7 SNPs [range 0-18 SNPs] in the same household; $p=0.027$ ). Pig isolates collected from unique farms were widely distributed across the phylogeny; the average pairwise SNP distance was 95.4 (range 3-224 SNPs).

We identified IEC in 20 isolates, which clustered within a closely related clade (Appendix 2 Figure). The IEC-harboring $\Phi S$ S3int prophages could be divided into 6 variants (I-VI) and 5 phylogenetic clusters (A-E) on the basis of their phylogenetic relationship, IEC type, and chromosomal integration site (Table 1; Figure 1). Each of the 6 ФSa3int variants was unique to isolates from a single household. The 6 households comprised 4 pig farm employees (ФSa3int-I-IV), a pig veterinarian (ФSa3int-V), and a mink farm employee (ФSa3int-VI), and their household contacts (Table $1)$. We also detected $\Phi S a 3 i n t-V I$ in 3 pig isolates that were closely related to the isolates from the mink farm employee's household; the prophage was furthermore highly similar to $\Phi$ Sa3int-V present in the isolate from the pig veterinarian but integrated into a different part of the chromosome (Table 1).

We identified the $\operatorname{tar} P$ gene in 45 isolates, which were more widely distributed across the phylogeny than the IEC-positive isolates (Appendix 2 Figure). Analysis of the genetically linked int genes showed that $\operatorname{tar} P$ was carried on 5 different prophages (ФSa1int, ФSa3int, ФSa7int, $\Phi$ Sa9int, and a prophage with an untypeable int gene hereafter referred to as ФUT1). BLASTN searches (https://blast.ncbi.nlm. nih.gov/Blast.cgi) showed that the int gene in $\Phi U T 1$ was $100 \%$ identical to the int gene in the bovine $S$. aureus phage $\Phi$ DW2 (GenBank accession no. KJ140076). We found $\Phi$ tarP-Sa9int exclusively in pig isolates $(\mathrm{n}=15)$, whereas the other $\operatorname{tar} P$-harboring prophages were identified in 25 human isolates from 16 households and in 5 pig isolates.

IEC was more prevalent in human isolates $(17 / 96$; $18 \%)$ than in pig isolates $(3 / 45 ; 6.7 \%)$, although the difference was not statistically significant (PR 2.66, $95 \%$ CI $0.90-8.26 ; p=0.12)$. By contrast, $\operatorname{tar} P$ was significantly less prevalent in human isolates $(25 / 96$; $26 \%$ ) than in pig isolates $(20 / 45 ; 44 \%$ ) (PR 0.59, 95\% CI 0.37-0.95; $\mathrm{p}=0.034)$. The IEC element in the 3 pig isolates was genetically linked to $\operatorname{tar} P$ on $\Phi$ Sa3int-V and $\Phi$ Sa3int-VI (Table 1; Appendix 2 Figure).

\section{Role of IEC and tarP during Household Transmission of LA-MRSA CC398 in North Denmark Region}

Household transmission of LA-MRSA CC398 was based on detection of secondary cases of colonization or infection with isolates that were closely related and genotypically indistinguishable from the isolate of the primary case. Households consisting of 1 person $(n=26)$ and households with $\geq 2$ persons reporting direct animal contact $(n=2)$ were excluded from both analyses. In addition, 1 household with a mixed population of IEC-positive and IEC-negative isolates was excluded from the corresponding analysis.

IEC-positive isolates were present in 14\% (5/36) of the eligible households. Secondary transmission occurred more often in IEC-positive households (4/5; $80 \%)$ than in IEC-negative households $(10 / 31 ; 32 \%)$, although the difference was not statistically significant (PR 2.48, 95\% CI 1.04-4.64; $p=0.064$ ). The proportion of secondary cases was significantly higher in IEC-positive households $(65 \% ; 11 / 17)$ than in IEC-negative households $(22 \% ; 16 / 74)$ (PR 2.99, 95\% CI 1.65-5.12; $p=0.0010)$. Isolates carrying $\operatorname{tar} P$ were present in $32 \%(12 / 37)$ of the eligible households. We

\begin{tabular}{|c|c|c|c|c|c|c|c|}
\hline Variant & $\begin{array}{l}\text { Phylogenetic } \\
\text { clustert }\end{array}$ & IEC type & Integration siteł & $\operatorname{tar} P$ & Isolate origin & Household ID & Farm ID \\
\hline I & $A$ & $\mathrm{~B}$ & 0723-0724 & - & $\begin{array}{c}1 \text { pig farm employee and } 4 \\
\text { household members }\end{array}$ & $\mathrm{H} 02$ & $\mathrm{~F} 01$ \\
\hline II & B & $\mathrm{E}$ & 2238 & - & $\begin{array}{l}1 \text { pig farm employee and } 1 \\
\text { household member }\end{array}$ & $\mathrm{H} 30$ & F01 \\
\hline III & C & B & $2059(h / b)$ & - & $\begin{array}{l}1 \text { pig farm employee and } 3 \\
\text { household members }\end{array}$ & H63 & F04 \\
\hline IV & $\mathrm{D}$ & B & 2591 (cidA) & - & 1 pig farm employee & H51 & F07 \\
\hline $\mathrm{V}$ & $\mathrm{E}$ & $\mathrm{F}$ & $2059(h / b)$ & + & 1 pig veterinarian & $\mathrm{H} 46$ & None \\
\hline \multirow[t]{4}{*}{ VI } & $\bar{E}$ & $\mathrm{~F}$ & 2644 & + & $\begin{array}{l}1 \text { mink farm employee and } 3 \\
\text { household members }\end{array}$ & $\mathrm{H} 49$ & $\mathrm{~F} 21$ \\
\hline & E & $\mathrm{F}$ & 2644 & + & Pig & NA & A \\
\hline & $E$ & $\mathrm{~F}$ & 2644 & + & Pig & NA & $B$ \\
\hline & $E$ & $\mathrm{~F}$ & 2644 & + & Pig & NA & C \\
\hline
\end{tabular}




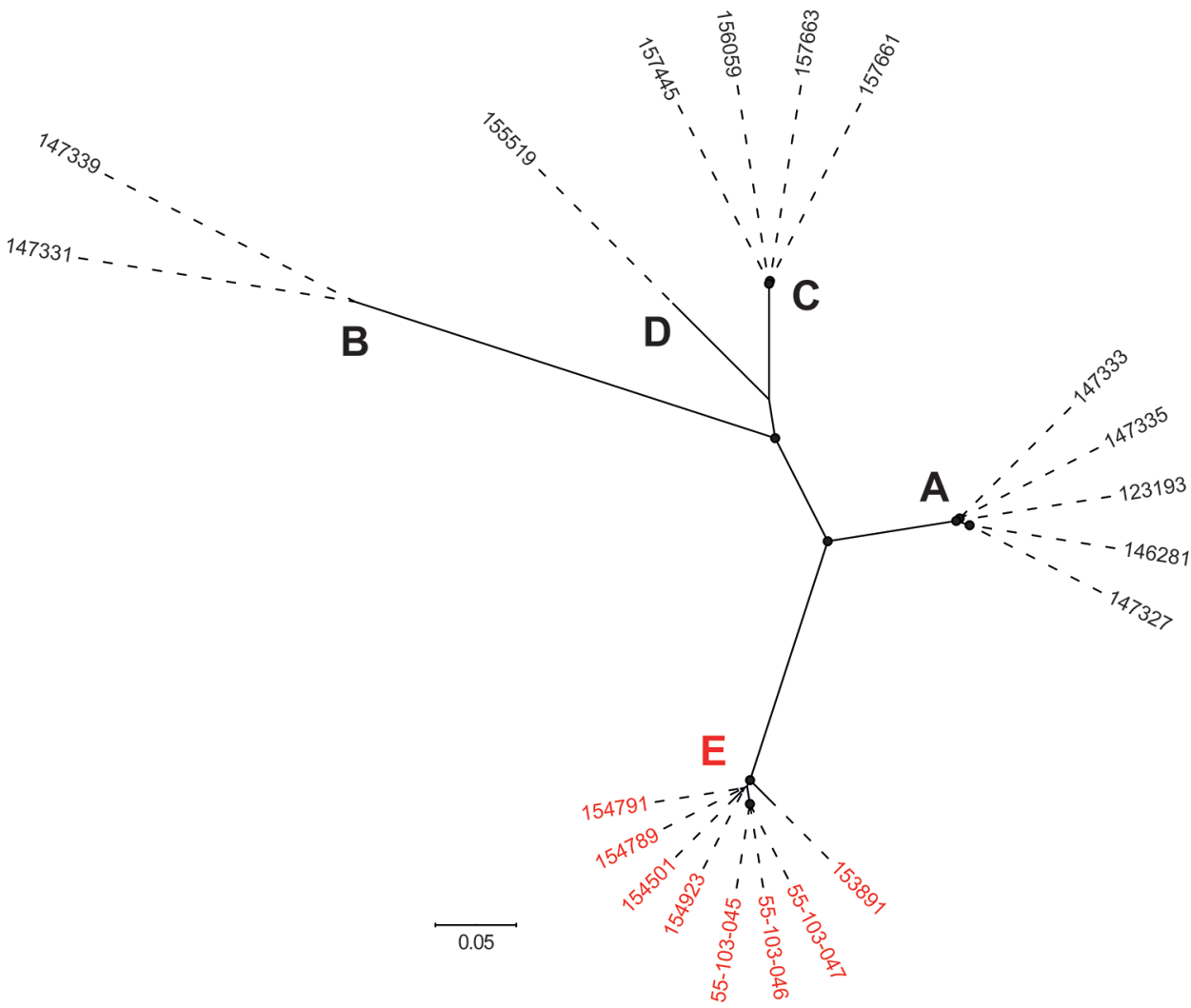

Figure 1. Maximum-likelihood phylogeny showing the genetic diversity of the 20 IECharboring $\Phi$ Sa3int prophages identified in livestockassociated methicillin-resistant Staphylococcus aureus CC398 isolates from North Denmark Region, Denmark. Capital letters indicate phylogenetic clusters (A-E). Red text indicates $\Phi$ Sa3int prophages harboring both IEC and tarP (cluster E). The phylogeny was estimated for 795 high-quality core SNPs. Filled circles at nodes indicate bootstrap values $>90 \%$. Scale bar represents number of nucleotide substitutions per variable site.

saw no differences in occurrence of secondary transmission between $\operatorname{tar} P$-positive households (5/12; $42 \%)$ and $\operatorname{tar} P$-negative households $(9 / 25 ; 36 \%)$ (PR $1.16,95 \%$ CI $0.47-2.55 ; p=1.00)$ or in the proportion of secondary cases between tarP-positive households $(10 / 33 ; 30 \%)$ and tarP-negative households (17/62; 27\%) (PR 1.28, 95\% CI 0.65- 2.42; p = 0.48). The average number of household contacts per household, excluding the primary case, did not differ significantly between IEC-positive (3.4; range 2-5) and IECnegative households $(2.4$; range $1-7 ; \mathrm{p}=0.17)$ or between $\operatorname{tar} P$-positive (2.8; range $1-7)$ and $\operatorname{tar} P$-negative households $(2.5$; range, $1-5 ; \mathrm{p}=0.62)$. These findings indicate that IEC, but not $\operatorname{tar} P$, facilitates household transmission of LA-MRSA CC398.

\section{Prevalence of IEC among LA-MRSA CC398 Isolates in Persons with No Livestock Contact}

We investigated whether IEC also plays a role during spread of LA-MRSA CC398 in the general population, on the assumption that persons with direct livestock contact serve as the source of transmission to their household contacts (i.e., persons with indirect livestock contact) and into the local community, through which the bacterium is transmitted into healthcare settings. The analysis included 1,545 isolates from patients in 4 groups: patients with direct livestock contact $(\mathrm{n}=727)$; patients with indirect livestock contact $(n=256)$; patients with $\mathrm{CO}$ infection $(\mathrm{n}=383)$; and patients with $\mathrm{HO} / \mathrm{HACO}$ infection $(\mathrm{n}=179)$. The results showed that the proportion of IEC-positive isolates increased along this hypothetical transmission chain, from 3.4\% in patients with direct contact to livestock to $6.3 \%$ (PR $1.82,95 \%$ CI $0.99-3.31 ; p=0.068)$ in patients with indirect contact to livestock, 7.1\% (PR 2.05, 95\% CI 1.213.46; $\mathrm{p}=0.010)$ in patients with CO infection, and $11 \%$ (PR 3.25, 95\% CI 1.85-5.65; $\mathrm{p}=0.0001$ ) in patients with $\mathrm{HO} / \mathrm{HACO}$ infection (Table 2). The excess number of clinical cases attributable to increased spread of IECpositive isolates ranged from 7 (2.8\%) among persons with indirect livestock contact to 14 in both the community (3.6\%) and healthcare (7.7\%) settings (Table 2). These findings demonstrate an association of IEC with increased human-to-human transmission and excess disease burden of LA-MRSA CC398.

If IEC-positive isolates become fixed (i.e., self-sustainable) in the general population, we expect their proportion to increase over time in patients with $\mathrm{CO}$ and $\mathrm{HO} / \mathrm{HACO}$ infections, compared with patients with direct contact to livestock. However, although the number of LA-MRSA CC398 infections increased in all 4 patient groups during 2007-2018 (Figure 2, 
Table 2. Patient characteristics and presence of IEC among livestock-associated methicillin-resistant Staphylococcus aureus CC398 cases and isolates, North Denmark Region, Denmark*

\begin{tabular}{|c|c|c|c|c|c|c|c|}
\hline Patient group & $\begin{array}{c}\text { No. } \\
\text { isolates }\end{array}$ & $\begin{array}{l}\text { Male:female } \\
\text { ratio }\end{array}$ & $\begin{array}{l}\text { Median age, y } \\
\text { (range) }\end{array}$ & $\%$ IEC & $\begin{array}{l}\text { No. IEC-related } \\
\text { excess cases } \\
\text { (\% all cases) }\end{array}$ & $\begin{array}{c}\text { Prevalence ratio } \\
(95 \% \mathrm{Cl})\end{array}$ & $p$ value \\
\hline \multicolumn{8}{|c|}{ Patients with livestock contact } \\
\hline Direct contact & 727 & 3.2 & $33(0-93)$ & 3.4 & Referent & Referent & Referent \\
\hline Indirect contact & 256 & 0.59 & $22(0-91)$ & 6.3 & $7(2.8)$ & $1.82(0.99-3.31)$ & 0.068 \\
\hline Patients with $\mathrm{CO}$ infection & 383 & 1.0 & $52(0-98)$ & 7.1 & $14(3.6)$ & $2.05(1.21-3.46)$ & 0.010 \\
\hline $\begin{array}{l}\text { Patients with } \mathrm{HO} / \mathrm{HACO} \\
\text { infection }\end{array}$ & 179 & 1.1 & $66(0-97)$ & 11 & $14(7.7)$ & $3.25(1.85-5.65)$ & 0.0001 \\
\hline
\end{tabular}

panel A), the prevalence ratio of IEC-positive isolates among patients with $\mathrm{CO}$ and $\mathrm{HO} / \mathrm{HACO}$ infections either decreased or remained relatively stable over the years (Figure 2, panel B).

\section{Discussion}

Successful spread of $S$. aureus in humans depends upon the bacterium's ability to survive and multiply in newly colonized persons. For example, $S$. aureus must compete with other bacteria and avoid the innate and adaptive immune defenses of the skin and nasal environments. In this study, we have shown that acquisition of IEC, but not $\operatorname{tar} P$, is associated with increased household transmission of LA-MRSA CC398 and excess spread into the community and healthcare settings. The findings should be interpreted with some caution because of the small number of households and cases analyzed, and more follow-up studies should be done to further evaluate the relative contribution of IEC and other risk factors, such as household size (13), to the spread of LA-MRSA CC398 in humans.

Animal and human studies have shown that IEC is ubiquitous in human S. aureus clones, whereas it is consistently absent in livestock-associated $S$. aureus clones $(9,10)$. Staphylococcal complement inhibitor and other IEC-encoded immune modulators have human-specific activities toward central components of the innate immune response, such as neutrophils and complements, and are produced in both healthy carriers and patients with S. aureus infection (14-16). Our findings suggest that IEC promotes survival of $S$. aureus in the human host, which in turn is expected to increase the likelihood of human-to-human transmission.

Nonetheless, the disease burden of IEC-positive LA-MRSA CC398 isolates in the community and healthcare settings remains relatively low in Denmark. We saw no suggestion that they will become fixed in the general population; van Alen et al. reached the same conclusion after finding a low, albeit slightly increasing, prevalence of IEC among LA-MRSA CC398 isolates from hospital patients during 2000-2015, ranging from $1.1 \%$ during $2000-2006$ to $3.9 \%$ during 2007 2015 (17). Research indicates several possible reasons why IEC-positive LA-MRSA CC398 isolates have not become self-sustainable in humans. First, our findings showed that the 20 IEC-positive isolates from North Denmark Region clustered within a closely related clade, indicating that not all LA-MRSA CC398 isolates have the same ability to acquire $\Phi$ Sa3int phages. Second, a recent study (18) has shown that LA-MRSA CC398 carries substitutions at the usual attachment site (attB) located within the hlb gene, which interferes with phage integration and might explain why $\Phi$ Sa3int phages often integrate into other genomic regions than
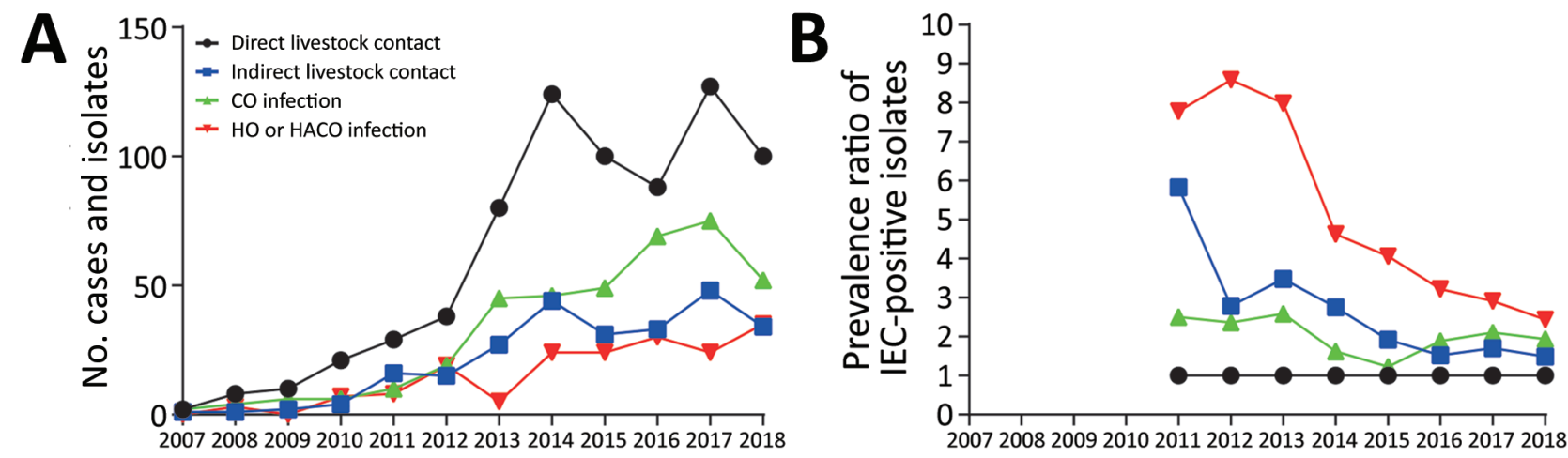

Figure 2. Temporal trends of livestock-associated methicillin-resistant Staphylococcus aureus infections in Denmark. A) Annual number of cases and isolates. B) Proportion of IEC-positive isolates in patients with indirect livestock contact, $\mathrm{CO}$, and $\mathrm{HO} / \mathrm{HACO}$ infection compared with patients who had direct contact with livestock. Data are shown as prevalence ratios (5-year rolling averages). CO, community-onset; HACO, healthcare-associated community-onset; $\mathrm{HO}$, healthcare-onset; IEC, immune evasion cluster. 
the $h l b$ gene in LA-MRSA CC398 through recombination between the phage attachment site (attP) and alternative attB sites, as demonstrated in our study and others (17-19). Third, it is possible that LA-MRSA CC398 has undergone other genetic changes, in addition to losing the IEC-harboring $\Phi$ Sa3int prophage during the human-to-animal host switch event, that are beneficial in the livestock reservoir but detrimental in the human host. For example, LA-MRSA CC398 isolates from Denmark have acquired several mobile genetic elements encoding resistance to a wide range of the most frequently used antimicrobial drugs in pigs, including $\beta$-lactams, aminoglycosides, macrolides, tetracyclines, and zinc (12), which are likely to exert a fitness cost outside the livestock reservoir (20). This possibility is supported by our recent finding that LA-MRSA CC398 isolates from hospital patients carry far fewer antimicrobial resistance genes of veterinary importance than LA-MRSA CC398 isolates from pigs (21).

The widespread distribution of IEC among human $S$. aureus clones, the phylogenetic clustering of IEC-harboring $\Phi$ Sa3int prophages in LA-MRSA CC398 isolates from the same household, and the low prevalence of IEC in LA-MRSA CC398 isolates from pigs suggest that acquisition of IEC by LA-MRSA CC398 mainly occurs through transfer of $\Phi$ Sa3int phages from human $S$. aureus donors circulating in households. In support of this view, we found little evidence for phage transfer or transmission of IECpositive isolates between pigs and humans. An exception was a possible transmission chain involving 3 IEC-positive isolates from pigs and 4 isolates from a mink farm employee and his household members and a possible phage transfer event between this cluster of isolates and an isolate from a pig veterinarian. Of note, the IEC-harboring $\Phi$ Sa3int prophage found in the 3 pig isolates also encoded TarP, thus raising the possibility that IEC can be passively maintained in the pig population by co-selection for other traits.

TarP-mediated protection against anti-WTA antibodies did not seem to influence household transmission of LA-MRSA CC398 in our study despite the fact that anti-staphylococcal antibodies are present at high levels in serum and nasal secretions of both persistent $S$. aureus carriers and noncarriers $(15,16)$. Instead, there is evidence that the much more widely distributed staphylococcal protein A (SpA), which is produced by all known human and livestock-associated $S$. aureus clones, is sufficient for escaping the adaptive immune response. SpA contains several immunoglobulin-binding domains capable of binding both the Fcy of IgG antibodies and the Fab of $\mathrm{V}_{\mathrm{H}}$ 3-idiotype antibodies, thereby limiting opsonophagocytosis and broad-spectrum antibody responses to other secreted and surface-bound antigens during $S$. aureus colonization and infection (22-30).

In summary, our study suggests that acquisition of IEC, but not $\operatorname{tar} P$, is associated with increased household transmission of LA-MRSA CC398 and spillover into the community and healthcare settings, which might also explain why IEC is widespread among human S. aureus clones. Despite these findings, the attributable disease burden remains relatively low in Denmark, and we found no evidence to suggest that they have become self-sustainable in the general population. However, the dynamic nature of $S$. aureus genome evolution and host adaptability, as documented here and elsewhere, underscores the need for continued surveillance at the human-animal interface to detect evolutionary as well as epidemiologic changes that affect public health.

\section{Acknowledgments}

We thank the staff at the local clinical microbiology departments and National Reference Laboratory for Antimicrobial Resistance for their invaluable contribution to this work.

This work was supported by the National Institute of Allergy and Infectious Diseases, National Institutes of Health (grant no. 1R01AI101371-01A1) for A.R.L., R.L.S., L.B.P., M.S., and J.L. and the Ministry of Environment and Food of Denmark through the Danish Agrifish Agency (grant no. 33010-NIFA-14-612) for R.N.S., A.R.L., R.L.S., and J.L.

\section{About the Author}

Dr. Sieber is a research associate in infectious diseases at Statens Serum Institut, Copenhagen. His research interests are the evolution of opportunistic pathogens and their epidemiology.

\section{References}

1. van Cleef BA, Monnet DL, Voss A, Krziwanek K, Allerberger F, Struelens M, et al. Livestock-associated methicillin-resistant Staphylococcus aureus in humans, Europe. Emerg Infect Dis. 2011;17:502-5. https://doi.org/10.3201/ eid1703.101036

2. Danish Integrated Antimicrobial Resistance Monitoring and Research Programme. DANMAP 2018: use of antimicrobial agents and occurrence of antimicrobial resistance in bacteria from food animals, food, and humans in Denmark. 2019 Sep [cited 2020 Mar 9]. https://www.danmap.org/-/media/arkiv/projekt-sites/ danmap/danmap-reports/danmap-2018/danmap_2018.pdf

3. Kinross P, Petersen A, Skov R, Van Hauwermeiren E, Pantosti A, Laurent F, et al.; European Human LA-MRSA Study Group. Livestock-associated meticillin-resistant Staphylococcus aureus (MRSA) among human MRSA 
isolates, European Union/European Economic Area countries, 2013. Euro Surveill. 2017;22:16-00696. https://doi.org/10.2807/1560-7917.ES.2017.22.44.16-00696

4. Larsen J, Petersen A, Sørum M, Stegger M, van Alphen L, Valentiner-Branth P, et al. Meticillin-resistant Staphylococcus aureus CC398 is an increasing cause of disease in people with no livestock contact in Denmark, 1999 to 2011. Euro Surveill. 2015;20:30021. https:/ / doi.org/10.2807/ 1560-7917.ES.2015.20.37.30021

5. Larsen J, Petersen A, Larsen AR, Sieber RN, Stegger M, Koch A, et al.; Danish MRSA Study Group. Emergence of livestock-associated methicillin-resistant Staphylococcus aureus bloodstream infections in Denmark. Clin Infect Dis. 2017;65:1072-6. https:// doi.org/10.1093/cid/cix504

6. Hetem DJ, Bootsma MC, Troelstra A, Bonten MJ. Transmissibility of livestock-associated methicillin-resistant Staphylococcus aureus. Emerg Infect Dis. 2013;19:1797-802. https://doi.org/10.3201/eid1911.121085

7. Thammavongsa V, Kim HK, Missiakas D, Schneewind O. Staphylococcal manipulation of host immune responses. Nat Rev Microbiol. 2015;13:529-43. https://doi.org/10.1038/ nrmicro3521

8. Bae T, Baba T, Hiramatsu K, Schneewind O. Prophages of Staphylococcus aureus Newman and their contribution to virulence. Mol Microbiol. 2006;62:1035-47. https:/ / doi.org/ 10.1111/j.1365-2958.2006.05441.x

9. Richardson EJ, Bacigalupe R, Harrison EM, Weinert LA, Lycett S, Vrieling M, et al. Gene exchange drives the ecological success of a multi-host bacterial pathogen. Nat Ecol Evol. 2018;2:1468-78. https://doi.org/10.1038/s41559-018-0617-0

10. Price LB, Stegger M, Hasman H, Aziz M, Larsen J, Andersen PS, et al. Staphylococcus aureus CC398: host adaptation and emergence of methicillin resistance in livestock. MBio. 2012;3:e00305-11. https://doi.org/10.1128/mBio.00305-11

11. Gerlach D, Guo Y, De Castro C, Kim SH, Schlatterer K, $\mathrm{Xu} \mathrm{FF}$, et al. Methicillin-resistant Staphylococcus aureus alters cell wall glycosylation to evade immunity. Nature. 2018;563:705-9. https:/ / doi.org/10.1038/ s41586-018-0730-x

12. Sieber RN, Skov RL, Nielsen J, Schulz J, Price LB, Aarestrup FM, et al. Drivers and dynamics of methicillinresistant livestock-associated Staphylococcus aureus CC398 in pigs and humans in Denmark. MBio. 2018;9:e02142-18. https:/ / doi.org/10.1128/mBio.02142-18

13. Di Ruscio F, Guzzetta G, Bjørnholt JV, Leegaard TM, Moen AEF, Merler S, et al. Quantifying the transmission dynamics of MRSA in the community and healthcare settings in a low-prevalence country. Proc Natl Acad Sci U S A. 2019;116:14599-605. https:/ / doi.org/10.1073/pnas.1900959116

14. Rooijakkers SH, Ruyken M, Roos A, Daha MR, Presanis JS, Sim RB, et al. Immune evasion by a staphylococcal complement inhibitor that acts on C3 convertases. Nat Immunol. 2005;6:920-7. https:// doi.org/10.1038/ni1235

15. Verkaik NJ, de Vogel CP, Boelens HA, Grumann D, Hoogenboezem T, Vink C, et al. Anti-staphylococcal humoral immune response in persistent nasal carriers and noncarriers of Staphylococcus aureus. J Infect Dis. 2009;199:625-32. https:// doi.org/10.1086/596743

16. Verkaik NJ, Lebon A, de Vogel CP, Hooijkaas H, Verbrugh HA, Jaddoe VW, et al. Induction of antibodies by Staphylococcus aureus nasal colonization in young children Clin Microbiol Infect. 2010;16:1312-7. https://doi.org/ 10.1111/j.1469-0691.2009.03073.x

17. van Alen S, Ballhausen B, Kaspar U, Köck R, Becker K. Prevalence and genomic structure of bacteriophage phi3 in human-derived livestock-associated methicillin-resistant
Staphylococcus aureus isolates from 2000 to 2015. J Clin Microbiol. 2018;56:e00140-18. https:// doi.org/10.1128/JCM.00140-18

18. Tang Y, Nielsen LN, Hvitved A, Haaber JK, Wirtz C, Andersen PS, et al. Commercial biocides induce transfer of prophage $\Phi 13$ from human strains of Staphylococcus aureus to livestock CC398. Front Microbiol. 2017;8:2418. https://doi.org/10.3389/fmicb.2017.02418

19. Kraushaar B, Hammerl JA, Kienöl M, Heinig ML, Sperling N, Dinh Thanh $\mathrm{M}$, et al. Acquisition of virulence factors in livestock-associated MRSA: lysogenic conversion of CC398 strains by virulence gene-containing phages. Sci Rep. 2017;7:2004. https://doi.org/10.1038/s41598-017-02175-4

20. Andersson DI, Hughes D. Antibiotic resistance and its cost: is it possible to reverse resistance? Nat Rev Microbiol. 2010;8:260-71. https:/ / doi.org/10.1038/nrmicro2319

21. Sieber RN, Larsen AR, Urth TR, Iversen S, Møller CH, Skov RL, et al. Genome investigations show host adaptation and transmission of LA-MRSA CC398 from pigs into Danish healthcare institutions. Sci Rep. 2019;9:18655. https://doi.org/10.1038/s41598-019-55086-x

22. Forsgren A. Significance of protein A production by staphylococci. Infect Immun. 1970;2:672-3. https:/ / doi.org/ 10.1128/IAI.2.5.672-673.1970

23. Björk I, Petersson BA, Sjöquist J. Some physiochemical properties of protein A from Staphylococcus aureus. Eur J Biochem. 1972;29:579-84. https://doi.org/10.1111/ j.1432- 1033.1972.tb02024.x

24. Potter KN, Li Y, Capra JD. Staphylococcal protein A simultaneously interacts with framework region 1 , complementarity-determining region 2, and framework region 3 on human $\mathrm{V}_{\mathrm{H}}$ 3-encoded Igs. J Immunol. 1996; 157:2982-8.

25. Graille M, Stura EA, Corper AL, Sutton BJ, Taussig MJ, Charbonnier JB, et al. Crystal structure of a Staphylococcus aureus protein A domain complexed with the Fab fragment of a human IgM antibody: structural basis for recognition of B-cell receptors and superantigen activity. Proc Natl Acad Sci U S A. 2000;97:5399-404. https://doi.org/10.1073/ pnas.97.10.5399

26. Goodyear CS, Silverman GJ. Death by a B cell superantigen: in vivo $\mathrm{V}_{\mathrm{H}}$-targeted apoptotic supraclonal $\mathrm{B}$ cell deletion by a staphylococcal toxin. J Exp Med. 2003;197:1125-39. https:// doi.org/10.1084/jem.20020552

27. Falugi F, Kim HK, Missiakas DM, Schneewind O. Role of protein $\mathrm{A}$ in the evasion of host adaptive immune responses by Staphylococcus aureus. MBio. 2013;4:e00575-13. https://doi.org/10.1128/mBio.00575-13

28. Pauli NT, Kim HK, Falugi F, Huang M, Dulac J, Henry Dunand C, et al. Staphylococcus aureus infection induces protein A-mediated immune evasion in humans. J Exp Med. 2014;211:2331-9. https:// doi.org/10.1084/jem.20141404

29. Sun Y, Emolo C, Holtfreter S, Wiles S, Kreiswirth B, Missiakas D, et al. Staphylococcal protein A contributes to persistent colonization of mice with Staphylococcus aureus. J Bacteriol. 2018;200:e00735-17. https://doi.org/10.1128/ JB.00735-17

30. Chen X, Sun Y, Missiakas D, Schneewind O. Staphylococcus aureus decolonization of mice with monoclonal antibody neutralizing protein A. J Infect Dis. 2019;219:884-8. https://doi.org/10.1093/infdis/jiy597

Address for correspondence: Jesper Larsen, Department of

Bacteria, Parasites and Fungi, Statens Serum Institut, Artillerivej 5, DK-2300 Copenhagen S, Denmark; email: JRL@ssi.dk 